\title{
A chamber and artificial dura method for long-term optical imaging in the monkey
}

\author{
Li Min Chen, Barbara Heider, Graham V. Williams, Francine L. Healy, \\ Benjamin M. Ramsden, Anna Wang Roe* \\ Section of Neurobiology, Yale University School of Medicine, 333, Cedar Street SHM-I412 New Haven, CT 06510, USA
}

Received 20 April 2001; received in revised form 30 August 2001; accepted 30 August 2001

\begin{abstract}
Optical imaging over extended periods of time in non-human primates presents serious challenges because the dura mater must be removed to expose the cortical surface. We present a novel nylon imaging chamber with a transparent artificial dura implant, which allows repeated, long-term optical recordings from the cortex. The cylinder of the chamber is inserted into a cranial trephination and held in place with a minimum of screws and acrylic cement. A round patch of artificial dura with a perpendicular wall protects the cortical surface and slows re-growth of dural tissue within the chamber. A cap, manufactured from the same material as the cylinder, is screwed into the chamber and seals it completely. Over a period of 1-4 months, the chamber required a minimum of maintenance and stayed infection-free without local antibiotic application. We repeatedly performed optical imaging in the same animal with the advantages of shortened preparation time. To permit precise alignment and comparison of maps obtained from different imaging sessions, we developed a program that calculated a 2-dimensional spatial transformation between maps of different magnifications, translations, and distortions. We suggest that these methods provide a practical solution to long-term optical imaging in the anesthetized or alert monkey. The exclusive use of non-metallic materials offers the benefit of a lighter and more compact implant, and the possibility to perform MRI scans after chamber implantation. (C) 2002 Elsevier Science B.V. All rights reserved.
\end{abstract}

Keywords: Optical imaging; Visual cortex; Non-human primate; Chronic implants; Dural substitute; Long-term recordings

\section{Introduction}

Intrinsic signal optical imaging has been used to map cortical functional organization at a submillimeter scale in the visual (e.g. Bartfeld and Grinvald, 1992; Bonhoeffer et al., 1995; Ts'o et al., 1990), auditory (Bakin et al., 1996; Spitzer et al., 2001), and somatosensory systems (Tommerdahl et al., 1998; Chen et al., 2001) of anesthetized animals. Optical imaging methods have also been used to study activation in awake, behaving animals (Grinvald et al., 1991; Vnek et al., 1999; Siegel et al., 1999; Slovin et al., 1999, 2000; Shtoyerman et al., 2000). Our goal in this paper was to develop a 'window on the brain' for use both in the anesthetized and in the

\footnotetext{
* Corresponding author. Tel.: + 1-203-737-5853; fax: + 1-203-7855263.

E-mail address: anna.roe@yale.edu (A.W. Roe).
}

awake, behaving monkey - a chronic chamber method that allows simple, infection-free maintenance.

To enable such a method, several issues must be dealt with. To maintain a clear, unobstructed view of the cortex, the skull and dura mater must be prevented from regrowing over the exposed cortex. Furthermore, as part of a 'healing response', cortex that is exposed for extended periods of time exhibits a growth of 'neo-membrane', a new membrane on the surface of the cortex that seriously degrades the quality of the optical signal and eventually prevents imaging altogether. Exposed cortex is also subject to added risk for infection and mechanical injury. Although in smaller animals (e.g. rats, tree shrews, cats) with thin dura, intrinsic optical signals can be imaged through the intact dura or even through the thinned bone (Cannestra et al., 1998; Bosking et al., 2000; Frostig et al., 1990), the thickness of the bone and native dura in old world primates 
makes this option impractical (Grinvald et al., 1991). Different methods using fiber optics have been explored in other species for recording deep structures in the brain (Rector et al., 1997, 1999). In this paper, we report an implant method that permits routine, repeated imaging sessions in the anesthetized monkey that may be applicable for imaging in the awake, behaving animal.

\section{Materials and methods}

\subsection{The chamber and artificial dura design}

The chamber is a prototype designed for electrophysiological and MRI recordings (G.V. Williams, unpublished observations; Fig. 1A). It is manufactured from FDA approved, hardened nylon (McMaster-Carr, Chicago, IL) and is thus lightweight and MRI-compatible. The top of the chamber is threaded on the inside so that a cap, made of the same material, screws into the chamber securely and provides a good seal (Fig. 1E). The bottom of the chamber consists of a number of flexible flanges spaced roughly $2-3 \mathrm{~mm}$ apart as shown in Fig. 1B.

The artificial dura is a transparent, soft thermoplastic polyurethane $\left(\right.$ Tecoflex $^{\circledR}$ ) as described by Sakas et al. (1990). This material is completely inert and does not produce reactive response from neural tissue. In previous surgical procedures, in which the dura is repeatedly opened and resutured over periods of a year or more, this material has proven safe and effective in preventing growth of adhesions between cortex and native dura (Roe and Ts'o, 1995, 1999; Ramsden et al., 2001).

We had previously performed long-term optical imaging in awake monkeys (six macaques and one squirrel monkey) that were implanted with regular metal chambers (cf. Vnek et al., 1999). In these experiments, we had initially used a flat round piece of the artificial dura. This usually kept the cortex clear for a few days before the native dura started to overgrow it. Later, we developed an artificial dura 'sandwich', which consisted of two round pieces, which were cauterized together around the center thus creating two flaps. After the durotomy, the edges of the native dura were tucked between the two flaps to prevent the dura from growing into the field of view. In two monkeys, this method was applied successfully but the insertion of the 'sandwich' proved to be rather difficult.

Subsequently, we developed the 'hat', which proved to be the most practical design. For the chronic chamber, the artificial dura is formed into a 'hat', consisting of a flat, round base to which a circular wall is attached by melting the lower rim with a cautery (Fig. 1C). The base of the hat serves to prevent growth of adhesions between the cortex and native dura and is sufficiently large to prevent dislodgment between the cortex and dura. The wall of the hat prevents growth of native dura into the chamber. The diameter of the wall matches the inner diameter of the chamber, and apposes the chamber wall to provide a reasonably good seal (Fig. 1D). This design was successfully tested in three monkeys that are reported here.

\subsection{Implantation of the chamber and artificial dura}

A precisely circular craniotomy was made by trephination over the cortical area to be imaged. The diameter of the craniotomy $(16 \mathrm{~mm})$ exactly matched the base of the chamber. The dura was resected and the dura edges cauterized. The artificial dura hat was then placed in the craniotomy so that the base was located under the remaining dura edges. The cylinder was inserted into the craniotomy by flexing back the flanges with a spatula, which secured the chamber within the craniotomy. To seal the implant, a thin layer of liquid acrylic cement was applied around the outer rim of the chamber. In lateral craniotomies, where the skull is more curved, screws were not necessary to hold the chambers (Fig. 1D and E, monkey 3). In medial locations, one or two screws were inserted into the skull around the chamber to prevent turning of the chamber within the craniotomy.

\subsection{Optical imaging with the chamber and artificial dura in the anesthetized animal}

After the monkey's head was secured in the stereotaxic frame, the outside of the chamber was cleaned with an antiseptic solution (chlorhexidine diacetate, Nolvasan; 3\% hydrogen peroxide). After having removed the cap, the inside of the chamber was rinsed with sterile saline. At times, a small amount of new dural growth emerged between the artificial dural wall and the chamber wall. This growth had a gelatinous consistency and was easily removed with either forceps or cotton swabs. To minimize pulsations for imaging, the chamber was filled with either agar $(4 \%)$ or agar with a transparent silicone wafer (RTV615; General Electric Company, Waterford, NY). In some cases, we imaged through the artificial dura alone. The total time required for chamber preparation was kept reasonably short (approximately 10-20 min). Following imaging, the agar or silicone wafer was removed, the chamber rinsed with sterile saline, and the cap replaced.

The state of the cortex within the chamber was observed regularly, at least once a week, and documented with either digital photos and/or optical imaging sessions. No antibiotics or antiseptic agents were applied within the chamber. If there were any signs of infection either in the chamber or at the wound margins, the animals were treated with systemic antibiotics 

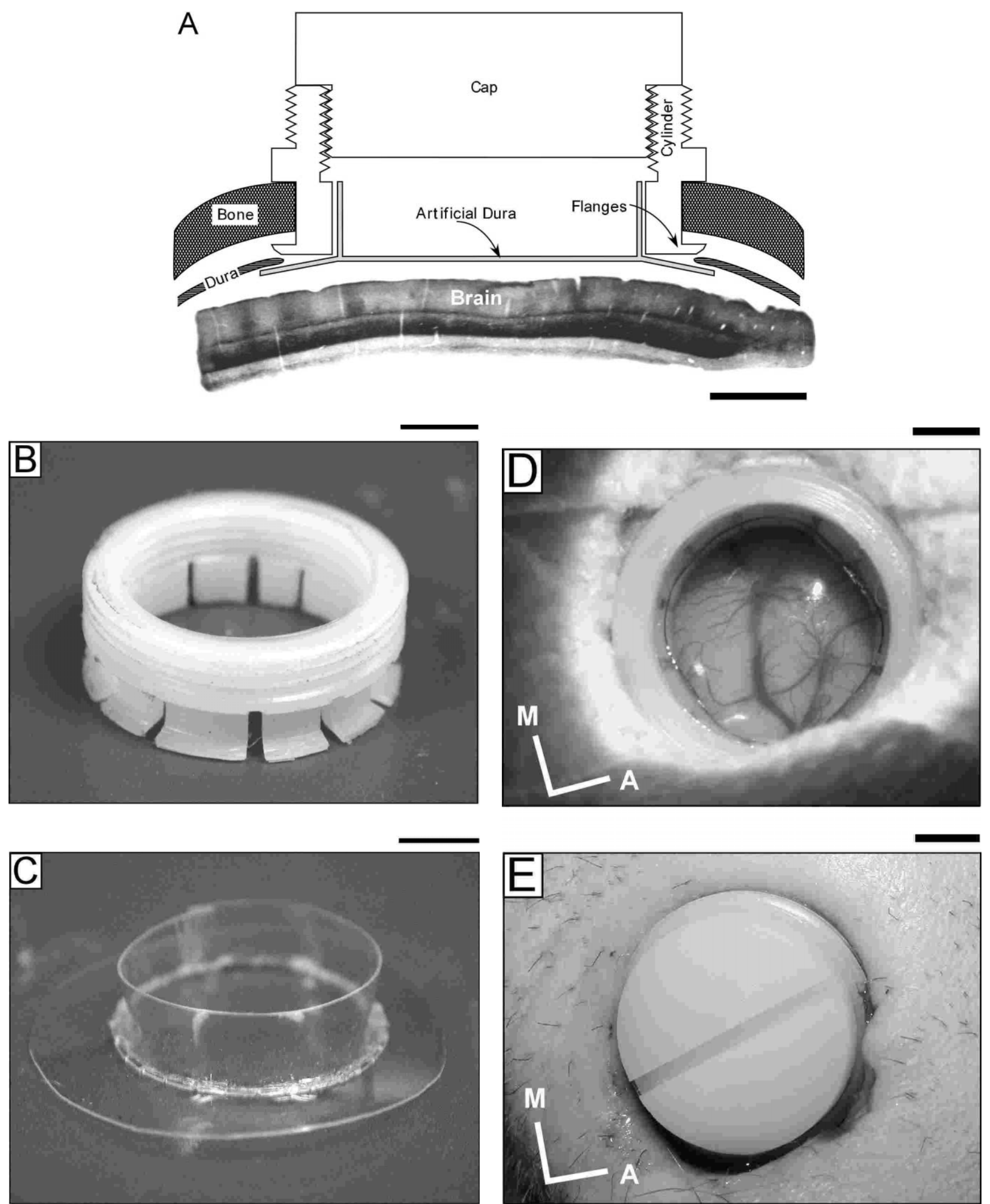

Fig. 1. The nylon chamber implant. Scale bar, $4 \mathrm{~mm}$. (A) Schematic overview of chamber implant showing the main components (cylinder, artificial dura hat and cap) and their placement within the trephination. (B) Photograph of the nylon cylinder showing the threaded outside and the flanges on the bottom with the slits to allow insertion into the trephination. (C) Photograph of the artificial dura 'hat'. (D) Open chamber in situ over the right hemisphere of monkey 3 exposing the lunate sulcus in the center of the chamber and the end of the inferooccipital sulcus in the anterior half of the chamber. (E) Appearance of same implant (monkey 3) in situ with cap closed. 
(enroflaxin, cefazolin). The monkeys' behavior, food intake, and weight were closely monitored to detect any possible discomfort as early as possible.

\section{Results}

We have studied three monkeys (Macaca fascicularis) using this chamber and artificial dura method. In monkeys 1 and 2, two chambers were implanted over each hemisphere - one medially over V1 and V2 and one laterally over V1, V2, and V4 (Gattass et al., 1981, 1988). In the third monkey, one chamber over the lateral location was implanted. Monkey 1 was sacrificed after a period of 4 months to observe the histological appearance of the tissue around the chamber region. Monkey 2 was sacrificed after 6 months.

\subsection{Cortical appearance}

Following implantation of the chamber and artificial dura, the state of the cortex remained healthy and constant in appearance over time. Fig. 2 displays four images from the right chamber of monkey 1 over a time course of 4 months (images shown are from days 21, 75, 91, and 117 post-implant). The cortex retained a normal appearance and blood vessel pattern (Fig. 2A$\mathrm{D}$, note similarity of major vessels). Our observations showed that there was an initial period, which lasted almost 1 month, without excessive growth of neo-membrane. Thus, we were able to image 21 days post implant without manipulations. After that time, the neo-membrane started covering the cortex and thus significantly degraded optical imaging quality.

\subsection{Neo-membrane management}

The neo-membrane, which is separate from the resected dural edges, initially appears as a thin transparent layer between cortex and artificial dura, often with new fine vasculature. This vasculature layer is clearly above the cortical vasculature when observed through the surgical microscope. In early stages of growth, this new tissue does not affect the imageability of the cor-
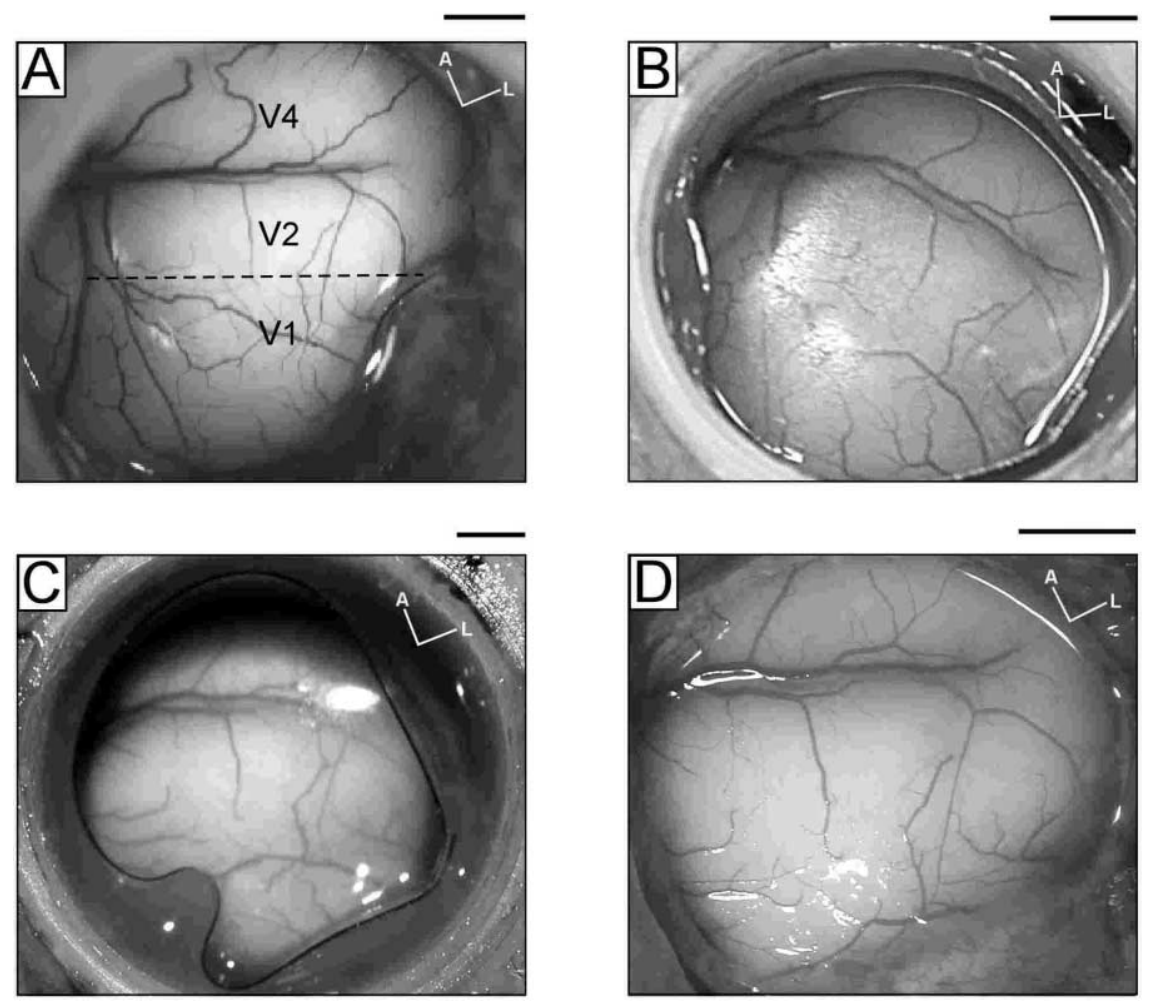

Fig. 2. Appearance of cortex (monkey 1) in the right hemisphere chamber at four different time points. Scale bar, $2 \mathrm{~mm}$. Visible in the anterior part of the chamber is the lateral end of the lunate sulcus. Areas V1 and V2 are located posterior to the sulcus, area V4 anterior. (A) Day 21 post implant, cortex looks clear and there are barely any signs of neo-membrane growth yet. (B) Day 75 post implant, after the neo-membrane was previously removed at day 49. There is no neo-membrane visible yet. (C) Day 91 post implant. The cortex looks rather clear; there is not much neo-membrane visible. (D) Day 117 post implant. In the first 3 images (A, B and C) the artificial dura is covering the cortical surface. The last image (D) was taken after the artificial dura and the cylinder were removed during the terminal experiment. The re-grown dura was resected at this time to expose the cortex for optical imaging. 

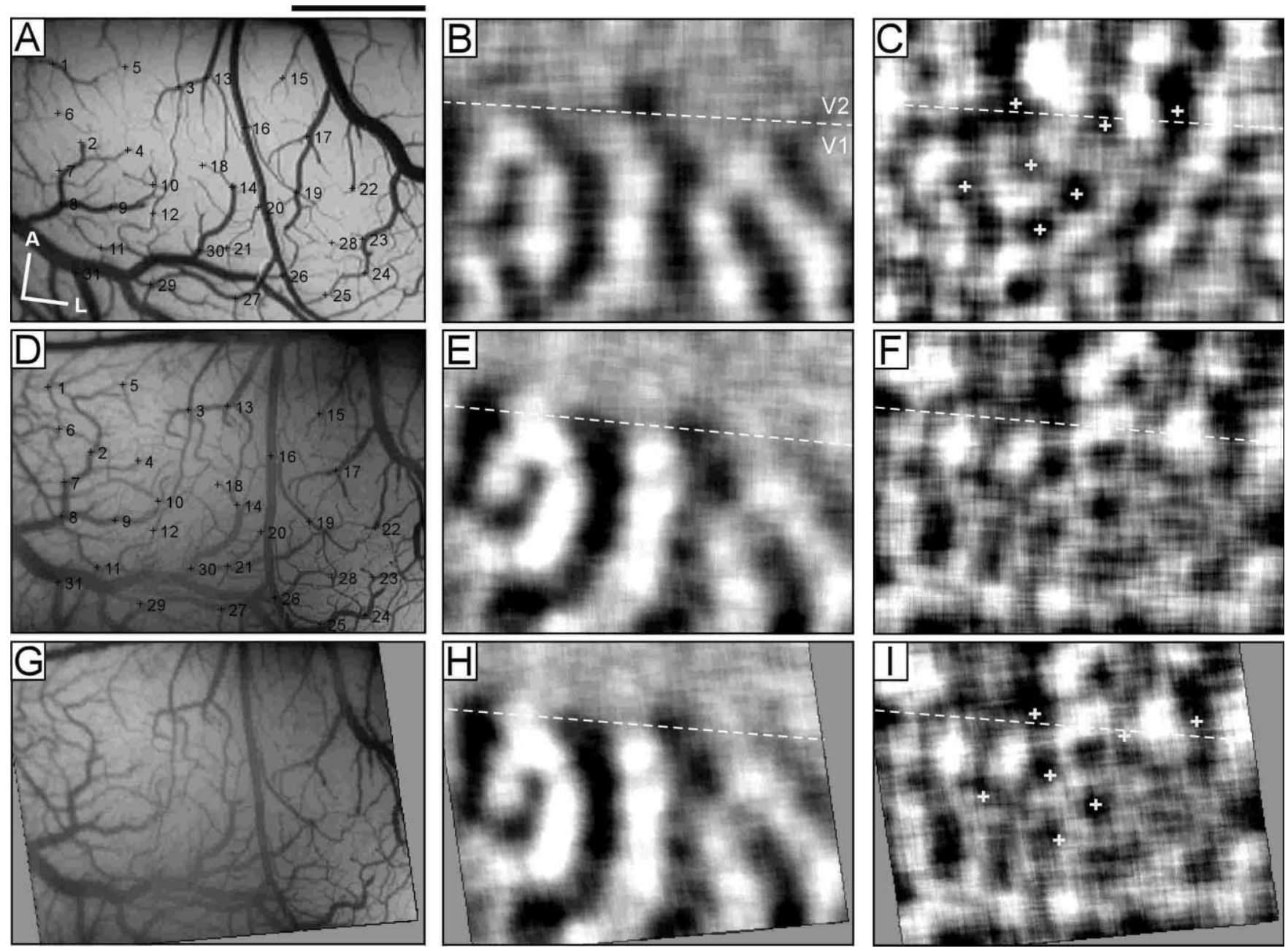

Fig. 3. Optical imaging of the medial parts of areas V1 and V2 in monkey 2 additionally showing maps from the alignment procedure. Scale bar, $2 \mathrm{~mm}$. The 31 black crosses with numbers in (A) and (D) indicate the alignment points for generating the transformation between images of different imaging sessions. (A) Blood vessel map as it appeared immediately after the craniotomy and durotomy before chamber implant. A temporary metal chamber was placed over the craniotomy and filled with silicone oil to stabilize the cortex. Images in (B) and (C) were acquired during this procedure with the animal being paralyzed (20 trials, low and high pass filtered). (B) Ocular dominance map in area V1 showing left eye (dark pixels) and right eye (light pixels). This map also marks the border between areas V1 and V2 (dashed line). (C) Orientation map with dark areas showing activity to horizontal (dark pixels) minus vertical (light pixels) drifting grating stimulation. Six orientation domains in V1 and V2 are marked with bright crosses for comparison with the transformed map shown in I. (D) Blood vessel map 60 days post implant through the artificial dura. Images in (E) and (F) were acquired in this procedure through artificial dura and agar (60 trials, low and high pass filtered) with paralysis. (E) Ocular dominance map (left eye, dark pixels; right eye, light pixels). (F) Orientation map (horizontal, dark pixels; vertical, light pixels) with drifting grating stimulation. (G-I) Transformed maps. (G) Blood vessels. (H) Ocular dominance (left eye minus right eye). (I) Orientation (horizontal minus vertical).

tex. Eventually this layer thickens, becomes opaque, and precludes imaging altogether. The growth of the neo-membrane is more rapid in cases of injury or abrasion to the cortex. If left untouched for a sufficient period of time (approximately 2-4 weeks), the neomembrane grows into a separable layer that can be easily resected without injury to the cortex. For such procedures, the artificial dura is easily removed and replaced. Attempts to remove neo-membrane before it has grown into a separate layer can result in injury to the cortex, as the membrane is rather delicate, disintegrates easily and tends to bleed at its initial stages.

\subsection{Repeatable functional maps}

To examine the functional state of the cortex over this period, we periodically imaged the area within the chamber. As shown in Fig. 3, ocular dominance (compare Fig. 3B and E) and orientation (compare Fig. 3C and F) maps maintained similar structure over a period of 60 days (monkey 2, images obtained under anesthesia and paralysis, at days 0 and 60 post implant). This monkey provided repeatable images on 13 separate imaging sessions (over a period of 6 months). The ability to obtain high quality maps had not diminished 
over this time and possibly can continue for a prolonged period of time.

\subsection{Image alignment}

Maps obtained from separate imaging sessions may differ slightly due to differences in imaging magnification, or from slight displacement or distortions of the cortex from day to day. To be able to precisely align and compare such images, we have developed an image alignment program that permits the summing of optical maps obtained from different sessions. As shown in Fig. $3 \mathrm{~A}$ and $\mathrm{D}$, this program uses matching landmark locations chosen from the blood vessel images. A 2-dimensional, linear spatial transformation is subsequently calculated between the two sets of landmark points that produces a minimal error difference. This transformation function is subsequently applied to functional maps. Due to local distortions, the fit may not be as good in some parts of the image as in others. Fig. 3 shows ocular dominance and orientation maps obtained from days 0 (Fig. 3B and C) and 60 post implant (Fig. 3E and F). Thirty-one corresponding points were chosen from the blood vessel maps (Fig. 3A and D) for calculating the transformation. This transformation was subsequently applied to images shown in Fig. 3D,
$\mathrm{E}$ and $\mathrm{F}$ to obtain images shown in Fig. $3 \mathrm{G}, \mathrm{H}$, and $\mathrm{I}$. For comparison, some domains are marked with crosses in Fig. 3C and I. As can be seen, the domains in $\mathrm{V} 1$ and $\mathrm{V} 2$ are in good correspondence. The local precision of such an alignment method is dependent on the density of the points chosen.

\subsection{Cortical stabilization}

The images acquired on day 0 (Fig. 3B and C) were obtained using silicone oil to stabilize the cortex, and those on day 60 (Fig. $3 \mathrm{E}$ and $\mathrm{F}$ ) were acquired with agar stabilization. In some cases (Fig. 4B), functional images were acquired only through the artificial dura without additional stabilization. This suggests that the artificial dura alone may provide sufficient stabilization for the acquisition of images, a possibility that could be quite advantageous during imaging in the awake animal.

In another case (monkey 1), similar images of ocular dominance were obtained over a period of 4 months. Fig. 4 illustrates two images acquired from the lateral chamber of this monkey on days 21 and 117 post implant. The images are similar in structure, although different in quality because the functional maps were obtained under different conditions. In Fig. 4B, imag-
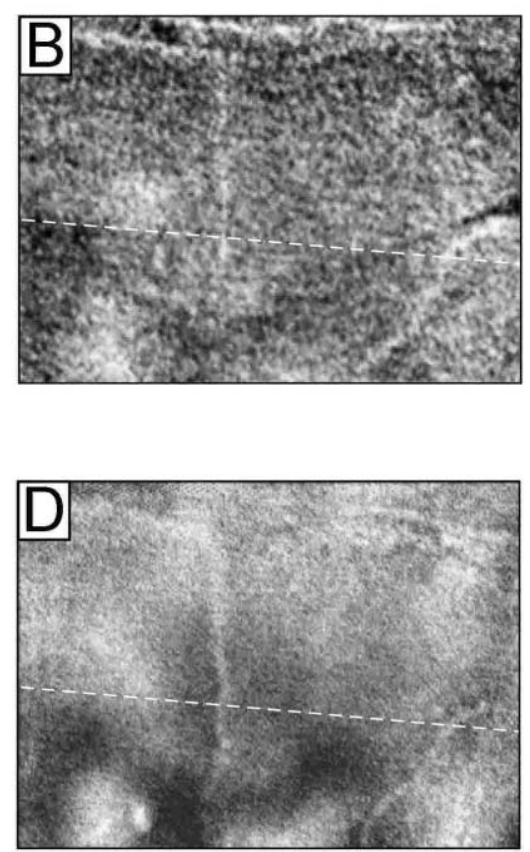

Fig. 4. Optical imaging of the lateral (foveal) parts of the visual cortex in monkey 1. Scale bar, $1 \mathrm{~mm}$. (A) Blood vessel map of the imaged area at day 21 post implant. (B) Ocular dominance map (left eye, dark pixels, minus right eye, light pixels) at day 21 post implant. The cortex was imaged through the artificial dura alone without silicone oil or agar stabilization, and the animal was not paralyzed (25 trials, no high/lowpass filtering). This decrease in signal-to-noise resulted in the poorer image quality in comparison to day 117 post implant. (C) Blood vessel map of same area at day 117 post implant. (D) Functional map showing ocular dominance activation (left eye minus right eye) at day 117 post implant. The upper end of the ocular dominance domains indicate the border between areas V1 and V2 (dashed line) located posterior to the lunate sulcus. In this session, the cortex was covered with agar for stabilization and the animal was paralyzed allowing image acquisition in synchrony with heart rate and respiration (25 trials, no high/lowpass filtering). 
ing was done without paralysis and further stabilization by agar or silicone oil. In Fig. 4D, imaging was done with paralysis and stabilized with agar. Thus, these images demonstrate long-term imageability with this method as well as approximate the decrease in signal to noise that would be expected in the awake animal (i.e. without paralysis and additional cortical stabilization).

\subsection{Anatomical and histological evaluation of brain tissue}

To obtain histological evaluation of cortical tissue health, monkey 1 was sacrificed after 4 months. After the brain was extracted and the dura removed, we observed that the part of the brain where the chambers were located was compressed along the flanges of the cylinder. This was mostly due to extensive growth of granulating tissue around the base of the two cylinders.

In the right hemisphere location, there were scarcely any differences between the cortex inside and outside the chamber. The cortical surface under the chamber looked very similar to fresh cortex. All layers were clearly discernible, and blobs could be seen in the cytochrome oxidase stained sections in area V1. However, in the left hemisphere location the cortical tissue within the chamber appeared degenerated. The cortex below the chamber was substantially thinner than normal and did not reveal the normal blob structure in V1 or staining of laminae $4 \mathrm{~A}$ and $4 \mathrm{C}$ when stained for cytochrome oxidase. We suspect that some initial damage to the cortex was the main reason for the degeneration of the cortical tissue in the left hemisphere chamber.

\section{Discussion}

We describe a new type of chamber implant designed for chronic long-term optical imaging in anesthetized and awake, behaving monkeys. We have shown that with this method the cortex remains healthy and imageable after 6 months (monkey 2) and likely longer following the initial durotomy and chamber implant. The chamber can be maintained for an extended period of time and functional images can be obtained repeatedly in the anesthetized animal. Moreover, we demonstrate a method for the precise alignment and comparison of maps obtained from different recording sessions. We suggest that these methods make optical imaging a practical and feasible method for studying cortical organization over an extended period of time in both the anesthetized and the awake, behaving animal.

Other investigators have also developed methods to address long-term imaging issues (cf. Grinvald et al., 1991; Vnek et al., 1999). Siegel et al. (1999) and Tanifuji and colleagues (Wang et al., 1998) are developing long-term methods for imaging parietal and inferotemporal cortex, respectively. Recently, Shtoyerman et al. (2000), with slightly different methods, have successfully demonstrated the ability to maintain imageable visual cortex in the awake monkey for a year or more. The authors showed that ocular dominance columns and orientation domains remained stable over a period of 8-9 months. Their artificial dura design is similarly hat-like, although made of a flexible silicone material, and the chamber is the standard metal optical chamber in contrast to the plastic snap-in chamber described here. With this design, Shtoyerman et al. (2000) do not report any growth of neo-membrane. There is, to our knowledge, no published discussion about management of the neo-membrane growth in chronic imaging implants, and no detailed comparison of different methods to maintain a long-term optical imaging window.

The important advantages of the current method concern the security, light weight, and low maintenance of the imaging chamber. Both the cylinder and the artificial dura hat are relatively easy to implant. In conventional implants, the metal chamber is attached to the surface of the skull and secured by inserting bone screws into the skull and applying dental cement to hold and seal the chamber. This design is usually stable and secure if a sufficient number of screws are inserted and the entire implant sealed with acrylic cement. Such extensive implants, however, can be a source of discomfort in young animals where the skull continues to grow underneath the rigid implant, and therefore, might cause tension. In addition, in some cases, the implant can be compromised by infection, tissue growth underneath the dental cement, or bone degeneration, leading to loosening of the chamber. With our current chamber design, the amount of cement required is minimal because the chamber grips the walls of the bone edge. One or two screws may be used to ensure that the chamber does not rotate within the craniotomy. The relatively small size and weight of the nylon chamber enables implanting of smaller animals (e.g. young monkeys, squirrel monkeys, cats), and easily accommodates more than one implant on the skull. In addition, fabrication with non-metallic materials permits post-implantation MRI scans for either diagnostic or experimental purposes.

Another advantage concerns the maintenance of chamber implants and the time and effort needed to prepare the chamber for imaging. Conventional chambers tested for long-term optical imaging were plagued by problems related to invading dural growth into the chamber (Roe et al., unpublished observations). This required constant clearing of dura, which often led to bleeding of dural edges, and stimulated more rapid neo-membrane growth. In addition, the dural manipulations required the animal to be slightly anesthetized each time (Vnek et al., 1999). With the current method, 
opening, cleaning and closing of the chamber is relatively rapid and there are hardly any tedious dura manipulations to prepare the cortex for imaging. As the cap tightly seals the chamber, we do not have to apply antiseptic or antibiotic substances locally. The artificial dura hat can be replaced easily without the risk of bruising the cortex and does not adhere to the neomembrane or to the cortex. This allows the removal of neo-membrane, the introduction of electrodes for cortical recordings, or the injection of anatomical tracers (not shown). Unlike other materials (e.g. silicone), our artificial dura is not penetrable by fine electrodes.

Methods to handle the growth of neo-membrane over the cortex underneath the artificial dura may be an area for further improvement. Observation of the tissue within the chambers over 4 months has shown that we were not able to prevent re-growth of neo-membrane over the cortex. It is possible that this neo-membrane is the initial stage of new dural growth, perhaps similar to that described in human neurosurgical literature (Berjano et al., 1999; Robertson and Menezes, 1997). The use of antimitotic agents or steroids has been suggested (Baker et al., 1999) to prevent extensive growth of dura in electrophysiological recordings, but this is not recommended when the cortex is exposed. We found that, provided that no cortical tissue damage occurs, the cortex can remain unobscured for weeks. However, we speculate that perhaps due to local tissue irritation caused by the edge of the artificial dura, a vascularized neo-membrane eventually develops. The use of an artificial dural hat with a larger diameter base might keep the neo-membrane growth away from the imaged region. Nonetheless, we found that this neo-membrane could be easily removed, exposing healthy cortex that provided reproducible, functional images. In addition to the dural re-growth, granulating tissue, which formed along the base of the cylinder, caused compression of the cortex along the lower rim of the chamber in some animals. Our imaging results, however, show that the functionality of the cortex was not compromised over the time period observed. Moreover, neurophysiological recordings in these chambers implanted over 1 year still produce consistent single unit responses (G.V. Williams and E. Procyk, unpublished observations).

In sum, we present a method for long-term optical imaging of cortical tissue following removal of native dura. We suggest that this method is practical and manageable for use in both the anesthetized and awake, behaving monkey.

\section{Acknowledgements}

We thank Robert Friedman, Daniel Y. Ts'o and Patricia S. Goldman-Rakic for helpful comments on the manuscript. We thank Neil Guo for developing the image alignment program. This work was supported by grants from NIH (EY11744), Packard Foundation, Browne-Coxe, and by a fellowship of the Swiss National Science Foundation (B.H., \# 8210-056578).

\section{Appendix A. Image alignment method}

Images (i.e. the measured images to be aligned or transformed) taken from different imaging sessions can be aligned with a common image (i.e. the reference image) through marks or reference points (on some clear well-defined features of blood vessel) that can be found on both reference and measured images. The coordinates of these reference points on the reference image are ( $X \mathrm{ref}, i, Y \operatorname{ref}, i)$, where $i=1,2, \ldots, N$ and $N$ is the total number of reference points. And, similarly, the coordinates of these reference points on the measured images are $(X \mathrm{~m}, i, Y \mathrm{~m}, i)$. The unit of these coordinates is pixel. There are the following relations between these two sets of coordinates:

$X \mathrm{ref}, i=\mathrm{f} x(X \mathrm{~m}, i, Y \mathrm{~m}, i)$,

and,

$Y \mathrm{ref}, i=\mathrm{f} y(X \mathrm{~m}, i, Y \mathrm{~m}, i)$,

where, $\mathrm{f} x$ and $\mathrm{f} y$ are polynomial functions of $X \mathrm{~m}, i$ and $Y \mathrm{~m}, i$. They can be expressed in:

$$
\begin{aligned}
X \mathrm{ref}, i= & A x X \mathrm{~m}, i+B x Y \mathrm{~m}, i+C x+D x X \mathrm{~m}, i^{2} \\
& +E x X \mathrm{~m}, i Y \mathrm{~m}, i+F x Y \mathrm{~m}, i^{2}+\ldots,
\end{aligned}
$$

and,

$$
\begin{aligned}
Y \mathrm{ref}, i= & A y X \mathrm{~m}, i+B y Y \mathrm{~m}, i+C y+D y X \mathrm{~m}, i^{2} \\
& +E y X \mathrm{~m}, i Y \mathrm{~m}, i+F y Y \mathrm{~m}, i^{2}+\ldots,
\end{aligned}
$$

where, $A x, B x, C x, D x, E x, F x, \ldots$ are constants of the transformation in $X$ axis or direction, and $A y, B y, C y$, $D y$, are the constants of the transformation in $Y$ axis or direction. These constants are to be found through the standard least squared method using the $N$ reference points. The first three terms in each equation are the linear terms, while the second three terms are the quadratic terms, cubic and higher order terms are not shown here, but can be included if found necessary.

The least squared solutions of these equations are proceeded iteratively. It is started with just the linear terms. From this initial solution, the constants can be determined. The coordinates of measured image $(X \mathrm{~m}, i$, $Y \mathrm{~m}, i)$ can then be transformed into the reference coordinate system, $\left(X^{\prime} \mathrm{m}, i, Y^{\prime} \mathrm{m}, i\right)$. The difference between $(X \mathrm{~m}, i, \quad Y \mathrm{~m}, i)$ and $\left(X^{\prime} \mathrm{m}, i, \quad Y^{\prime} \mathrm{m}, i\right), \quad(X \mathrm{~m}, i-$ $\left.X^{\prime} \mathrm{m}, i, Y \mathrm{~m}, i-Y^{\prime} \mathrm{m}, i\right)$, are the residual errors of the reference points. Residual graphs, $X \mathrm{~m}, i-X^{\prime} \mathrm{m}, i$ versus $X \mathrm{~m}, i, X \mathrm{~m}, i-X^{\prime} \mathrm{m}, i$ versus $Y \mathrm{~m}, i, Y \mathrm{~m}, i-Y^{\prime} \mathrm{m}, i$ versus $X \mathrm{~m}, i$, and, $Y \mathrm{~m}, i-Y^{\prime} \mathrm{m}, i$ versus $Y \mathrm{~m}, i$, are plotted. Based on the graphs, two major actions are taken. 
First, outliers of the reference points with large residuals, e.g. $\left(X \mathrm{~m}, i-X^{\prime} \mathrm{m}, i\right)>3 \sigma_{x}\left(\sigma_{x}\right.$ is the standard error of the least squared solution in the $X$ direction) or $\left(Y \mathrm{~m}, i-Y^{\prime} \mathrm{m}, i\right)>3 \sigma_{y}\left(\sigma_{y}\right.$ is the standard error of the least squared solution in the $Y$ direction), if there is any, are excluded from the following iteration of solutions. Second, distribution patterns of the residual points are carefully examined to see if quadratic and higher order terms of the polynomial equations need to be included in the following iterations. If the points are distributed randomly above and below the zero residual line, the transformation is good enough and the two sets of the constants are used for the alignment of the whole measured image.

From our experiment and actual transformation, we found that the linear transformation is normally accurate enough and higher order terms are not necessary. The standard error of the transformation, $\sigma_{x}$ and $\sigma_{y}$, are mostly at the sub-pixel level, or around \pm 0.1 to \pm 0.5 pixels, depending on the availability of welldefined sharp blood vessel features on the maps. This is accurate enough for the measured images to be transformed (including translation, rotation, and scaling) into the same position, orientation and scale of the common reference image.

\section{References}

Baker SN, Philbin N, Spinks R, Pinches EM, Wolpert DM, MacManus DG, Pauluis Q, Lemon RN. Multiple single unit recording in the cortex of monkeys using independently moveable microelectrodes. J Neurosci Methods 1999;94:5-17.

Bakin JS, Kwon MC, Masino SA, Weinberger NM. Suprathreshold auditory cortex activation visualized by intrinsic signal optical imaging. Cereb Cortex 1996;6:120-30.

Bartfeld E, Grinvald A. Relationships between orientation-preference pinwheels, cytochrome oxidase blobs, and ocular dominance columns in primate striate cortex. Proc Natl Acad Sci USA 1992;89:11905-9.

Berjano R, Vinas FC, Dujovny M. A review of dural substitutes used in neurosurgery. Crit Rev Neurosurg 1999;9:217-22.

Bonhoeffer T, Kim DS, Malonek D, Shoham D, Grinvald A. Optical imaging of the layout of functional domains in area 17 and across the area $17 / 18$ border in cat visual cortex. Eur $\mathbf{J}$ Neurosci 1995;7:1973-88.

Bosking WH, Kretz R, Pucak ML, Fitzpatrick D. Functional specificity of callosal connections in tree shrew striate cortex. J Neurosci 2000;20:2346-59.

Cannestra AF, Pouratian N, Shomer MH, Toga AW. Refractory periods observed by intrinsic signal and fluorescent dye imaging. J Neurophysiol 1998;80:1522-32.

Chen LM, Friedman RM, Ramsden BM, LaMotte RH, Roe AW. Fine-scale organization of primary somatosensory cortex (area $3 b)$ in the squirrel monkey revealed with intrinsic optical imaging. J Neurophysiol 2001, in press.
Frostig RD, Lieke EE, Ts'o DY, Grinvald A. Cortical functional architecture and local coupling between neuronal activity and the microcirculation revealed by in vivo high-resolution optical imaging of intrinsic signals. Proc Natl Acad Sci USA 1990;87:6082-6.

Gattass R, Gross CG, Sandell JH. Visual topography of V2 in the macaque. J Comp Neurol 1981;201:519-39.

Gattass R, Sousa APB, Gross CG. Visuotopic organization and extent of V3 and V4 of the Macaque. J Neurosci 1988;8:1831-45.

Grinvald A, Frostig RD, Siegel RM, Bartfeld E. High-resolution optical imaging of functional brain architecture in the awake monkey. Proc Natl Acad Sci USA 1991;88:11559-63.

Ramsden BM, Hung CP, Roe AW. Real and illusory contour processing in Area V1 of the primate: a cortical balancing act. Cereb Cortex 2001;11:648-65.

Rector DM, Poe GR, Redgrave P, Harper RM. A miniature CCD video camera for high-sensitivity light measurements in freely behaving animals. J Neurosci Methods 1997;78:85-91.

Rector DM, Rogers RF, George JS. A focusing image probe for assessing neural activity in vivo. $\mathbf{J}$ Neurosci Methods 1999;91:135-45.

Robertson SC, Menezes AH. Hemorrhagic complications in association with silastic dural substitute: pediatric and adult case reports with a review of the literature. Neurosurgery 1997;40:201-6.

Roe AW, Ts'o DY. Visual topography in primate V2: multiple representation across functional stripes. J Neurosci 1995;15:3689715.

Roe AW, Ts'o DY. Specificity of color connectivity between primate V1 and V2. J Neurophysiol 1999;82:2719-30.

Sakas DE, Charnvises K, Borges LF, Zervas NT. Biologically inert synthetic dural substitutes. Appraisal of a medical-grade aliphatic polyurethane and a polysiloxane-carbonate block copolymer. J Neurosurg 1990;73:936-41.

Shtoyerman E, Arieli A, Slovin H, Vanzetta I, Grinvald A. Longterm optical imaging and spectroscopy reveal mechanisms underlying the intrinsic signal and stability of cortical maps in V1 of behaving monkeys. J Neurosci 2000;20:8111-21.

Siegel RM, Phinney RE, Turner JA. Optical imaging of angle-of-gaze tuning in parietal area $7 \mathrm{a}$ of the behaving monkey. Soc Neurosci Abstr 1999;25:1547.

Slovin H, Arieli A, Hildesheim R, Grinvald A. Long-term voltagesensitive dye imaging in the behaving monkey. Soc Neurosci Abstr 1999;25:784.

Slovin H, Arieli A, Grinvald A. Voltage-sensitive dye imaging reveals that behavioral context affects population activity in early visual cortex. Soc Neurosci Abstr 2000;26:1082.

Spitzer MW, Calford MB, Clarey JC, Pettigrew JD, Roe AW. Spontaneous and stimulus-evoked intrinsic optical signals in primary auditory cortex of the cat. J Neurophysiol 2001;85:1283-98.

Tommerdahl M, Delemos KA, Favorov OV, Metz CB, Vierck CJ Jr, Whitsel BL. Response of anterior parietal cortex to different modes of same-site skin stimulation. J Neurophysiol 1998;80:3272-83.

Ts'o DY, Frostig RD, Lieke EE, Grinvald A. Functional organization of primate visual cortex revealed by high resolution optical imaging. Science 1990;249:417-20.

Vnek N, Ramsden BM, Hung CP, Goldman-Rakic PS, Roe AW. Optical imaging of functional domains in the cortex of the awake and behaving monkey. Proc Natl Acad Sci USA 1999;96:405760.

Wang G, Tanifuji M, Tanaka K. Functional architecture in monkey inferotemporal cortex revealed by in vivo optical imaging. Neurosci Res 1998;32:33-46. 